\title{
Inverse differentiability contractors and equations in Banach spaces
}

\author{
by \\ M. ALTMAN (Baton Rouge, La.)
}

\begin{abstract}
Alstract. Introducing the notions of inverse differentiability and contractors wo obtain a unified approach to various different in character iteration procedures including the Banach fixed point principle and the Newton-Kantorovich method. An application to evolution equations and a generalization of Krasnoselskii's fixed point theorem are also given.
\end{abstract}

\section{Contents}

Introduction ........................ 1

1. Inverse derivatives . . . . .................. . . 2

2. Iteration procedures with inverse derivatives ........... . 3

3. Contractors . . . . . . . . . . . . . . . . . . . . . 5

4. Implicit functions using contractors . . . . . . . . . . . . . . 6

5. A generalization of the Banach contraction principle . . . . . . . . . . 7

6. Nonlinar evolution equations, a generalization of the Piccard theorem in Banach spaces ........................ 9

7. A coincidence point theorem and a generalization of Krasnoselskii's fixed point theorem ........................ . 10

8. Stationary points of nonlinear functionals . . . . . . . . . . . . . 11

9. Various iteration procedures as special cases of the contractor method . . . 12 References. . . . . . . . . . . . . . . . . . . . . 15

Introduction. Recently Nashed [11] gave a systematic and very comprehensive exposition of abstract differential calculus in normed and topological linear spaces showing the important role of differentials in nonlinear functional analysis. In this paper we are concerned with one aspect of this concept as a tool to investigate iteration procedures for solving equations in Banach spaces. It is the nature of many applied problems leading to operator equations that the inverse operator is required to exist and to be even continuous. Thus, the regularity conditions are in the space of images. For this reason for instance the iteration procedure in the well known implicit function theorem uses the inverse of the Fréchet (Gâteaux) derivative. The same fact is seen in the NewtonKantorovich procedure (see [5], [6], [12]). This observation leads us to an independent definition of derivative such that can be 
used in place of the inverse of the derivative. Moreover, it turns out that for the same purposes much less can be required and what is the concept of a contractor. As a matter of fact if the mapping is a contraction itself then the contractor exists and is simply the identity mapping. The concept of a contractor generalizes the notion of an inverse derivative. The iteration procedure based on this concept is actually a perturbation method of the independent vector of the domain of the operator.

1. Inverse derivatives. Let $P: X \rightarrow Y$ be a nonlinear operator from a Banach space $X$ to a Banach space $Y$. Consider the difference $\mathrm{P}(x+h)$ $-P x=Q(x) h$ and suppose that $\Gamma(x)$ is a linear bounded operator associated. with $x \in X$ acting from $Y$ to $X$, i. e. $\Gamma(x): Y \rightarrow X$.

If $\Gamma(x)$ has the property: for $y \in X$

$$
\|y\|^{-1}\|Q(x) \Gamma(x) y-y\| \rightarrow 0 \text { as } y \rightarrow 0,
$$

then $\Gamma(x)$ is called the inverse derivative at $x$ of $P$. Condition (1.1) can be written in the form

$$
\|y\|^{-1}\|P(x+\Gamma(x) y)-P x-y\| \rightarrow 0 \text { as } y \rightarrow 0 .
$$

Properties of inverse derivatives.

(i) If $\Gamma(x)$ exists, then $d /\left.d t P(x+t \Gamma(x) y)\right|_{t=0}=y$, i e.P has a directional derivative in the direction $\Gamma(x) y$.

Property (i) shows that

(ii) $\Gamma(x) y=0$ implies $y=0$, i. e. $\Gamma(x)$ is a one-to-one mapping.

(iii) If the Fréchet derivative $P^{\prime}(x)$ exist, then $P^{\prime}(x)$ is an extension of $[\Gamma(x)]^{-1}$.

(iv) If $\left[P^{\prime}(x)\right]^{-1}$ exists, then $\Gamma(x)$ is uniquely defined and $\Gamma(x)$ $=\left[P^{\prime}(x)\right]^{-1}$. $=\Gamma(x)$

(v) If $\Gamma(x)$ is onto, then $P^{\prime}(x)$ exists and has the inverse $\left[P^{\prime}(x)\right]^{-1}$

Proposition. If $P^{\prime}(x)$ and $\Gamma(x)$ exist, then $P^{\prime}(x)$ maps $X$ onto $Y$ and there is a projection of $X$ onto the kernel of $P^{\prime}(x)$, i. e. $X=N \oplus X_{N}$ (direct sum), where $N=\left[h: P^{\prime}(x) h=0, h \in X\right]$ and $X_{N}$ is the range of $I^{\prime}(x)$.

Proof. Since $P^{\prime}(x) \Gamma(x) y=y$ for arbitrary $y \in Y$, by (iii), $P^{\prime}(x)$ is onto. It is easily seen that $X_{N}$ is closed. In fact, $\Gamma(x) y_{n} \rightarrow x_{0}$ implies $y_{n}=P^{\prime}(x) \Gamma(x) y_{n} \rightarrow P^{\prime}(x) x_{0}=y_{0}$, by (iii). Hence, $\Gamma(x) y_{n} \rightarrow \Gamma(x) y_{0}=x_{0}$.

For an arbitrary $h$ of $X$ let $y=P^{\prime}(x) h$ and $h_{p}=\Gamma(x) y$. Then, by (iii), $P^{\prime}(x) h_{p}=[\Gamma(x)]^{-1} h_{p}=y$. Hence, $P^{\prime}(x)\left(h-h_{p}\right)=0$, i. e. $h-h_{p} \epsilon N$, where $h_{p} \in X_{N}=\Gamma(x)(Y)$. Clearly, $N \cap X_{N}=\{0\}$.

Note that this concept of an inverse derivative is considered in the strong (Fréchet) sense. However, it is also possible to introduce the notion of an inverse derivative in a weaker sense of Gâteaux.
Remark 1.1. Calling $\Gamma(x)$ a right inverse derivative we could also introduce in a similar way a left inverse derivative $\Gamma(x)$ using the following formula in place of (1.1):

$$
\|h\|^{-1}\|\Gamma(x) Q(x) h-h\| \rightarrow 0 \quad \text { as } h \rightarrow 0, h \in X .
$$

2. Iteration procedures with inverse derivatives. Our problem is to find a solution to the operator equation

$$
P x=0,
$$

where $P: X \rightarrow Y, X$ and $Y$ are Banach spaces. We assume the existence of inverse derivatives $\Gamma(x)$ of $P$ in a neighborhood $S\left(x_{0}, r\right)=\left[x:\left\|x-x_{0}\right\|\right.$ $\leqslant r, x \in X]$, where $x_{0}$ is a given approximate solution to (2.1). For solving (2.1) we use the following iteration procedure:

$$
x_{n+1}=x_{n n}-\Gamma\left(x_{n}\right) P x_{n}, n=0,1,2, \ldots
$$

The following theorem gives sufficient conditions for the convergence of the iteration procedure (2.2) to a solution of equation (2.1).

THEOREM 2.1. Suppose that there exist positive numbers $0<q<1$, $r, \eta$ and $B$ such that the inverse derivative satisfies the uniformity condition. (2.3) $\|y\|^{-1}\|P(x+\Gamma(x) y)-P x-y\| \leqslant q \quad$ for $x \in S\left(x_{0}, r\right)$ and $\|y\| \leqslant \eta$.

$$
\begin{gathered}
\|\Gamma(x)\| \leqslant B \quad \text { for } x \in S\left(x_{0}, r\right) . \\
\left\|P\left(x_{0}\right)\right\| \leqslant \eta,
\end{gathered}
$$$$
B \eta(1-q)^{-1} \leqslant r \text {. }
$$

(2.7) $P$ is closed on $S\left(x_{0}, r\right)$.

Then there exists a solution $x^{*} \in S\left(x_{0}, r\right)$ and the sequence of $x_{n}$ defined by (2.2) converges toward $x^{*}$, i. e.

and

$$
x_{n} \rightarrow x^{*}, \quad P x^{*}=0, \quad x^{*} \in \mathcal{S}\left(x_{0}, r\right)
$$

$$
\left\|x_{n}-x^{*}\right\| \leqslant B q^{n}(r-q)^{-1} .
$$

Proof. Putting $y=-P x_{n}$ in (2.3) we obtain

$$
\left\|P x_{n+1}\right\| \leqslant q\left\|P x_{n}\right\|, \quad n=0,1,2, \ldots
$$

Hence, it follows from (2.2) and (2.4)

$$
\left\|x_{n+1}-x_{n}\right\| \leqslant B\left\|P x_{n}\right\| \leqslant B \eta q^{n} .
$$

By induction it is easy to see that $x_{n} \in S\left(x_{0}, r\right)$ for $n=0,1,2, \ldots$ Thus the sequence of $x_{n}$ converges toward some $x^{*} \in S\left(x_{0}, r\right)$. Since $P x_{n} \rightarrow \mathbf{0}$, by (2.9), and $P x$ is closed in $S\left(x_{0}, r\right)$, it follows that $P x^{*}=0$. The error estimate (2.8) results from (2.10) in the usual way. 
Remark 2.1. If $\Gamma(x)$ is onto, then the iteration procedure (2.2) becomes the well known Newton-Kantorovich method (see [5], [6], [12])

$$
x_{n+1}=x_{n}-\left[P^{\prime}\left(x_{n}\right)\right]^{-1} P x_{n} .
$$

In this case under the hypotheses of Theorem 2.1 the solution $x^{*}$ is unique in $S\left(x_{0}, r\right)$ if, in addition, condition (2.3) is satisfied for all $y \in Y$ such that $\|\Gamma(x) y\| \leqslant 2 r$, where $x \in S\left(x_{0}, r\right)$. This results from the following inequality

$$
(1-q)\|y\| \leqslant\|P(x+\Gamma(x) y)-P x\|
$$

obtained from (2.3). For if $x^{*}, x^{* *} \in S\left(x_{0}, r\right)$ are two solutions, then $x^{*}$ can be written as $x^{* *}=x^{*}+\Gamma\left(x^{*}\right) y$ and we have

$$
\left\|\Gamma\left(x^{*}\right) y\right\|=\left\|x^{* *}-x^{*}\right\| \leqslant 2 r
$$

and we can apply the inequality (2.12).

Consider now the equation $F(x)=0$, where $F: X \rightarrow R$ (reals) is a nonlinear functional on $X$. If the gradient $F^{\prime}(x)$ exists and $F^{\prime}(x) h \neq 0$, where $h \epsilon X$, then we have $t^{-1}\left|F\left(x+t\left[F^{\prime}(x) h\right]^{-1} h\right)-F(x)-t\right| \rightarrow 0$ as $t \rightarrow 0$, where $t \in R$, i. e. according to (1.2), $\Gamma(x) t=t\left[F^{\prime}(x) h\right]^{-1} h: R \rightarrow X$ is an inverse derivative of $F$ at $x$, if $h$ is fixed and $F^{\prime}(x) h \neq 0$.

Consider now the following generalization of Newton's method for nonlinear functionals which is given in [1].

$$
x_{n+1}=x_{n}-\left[F^{\prime}\left(x_{n}\right) h_{n}\right]^{-1} F^{\prime}\left(x_{n}\right) h_{n} ; \quad x_{n}, h_{n} \in X .
$$

Remark 2.2. The generalized Newton method (2.13) for nonlinear functionals in also a special case of an iteration procedure (2.2) with inverse derivatives.

Although the methods (2.11) and (2.13) are entirely different, both can be considered as particular cases of the procedure (2.2).

We say that $\Gamma\left(x_{0}\right)$ is a uniform inverse derivative of $P$ at $x_{0}$ if the following condition is satisfied (provided $\Gamma\left(x_{0}\right)$ is an inverse derivative)

$$
\|y\|^{-1}\left\|P\left(x+\Gamma\left(x_{0}\right) y\right)-P x-y\right\| \leqslant q
$$

for $\|y\| \leqslant \eta$ and for $x$ in some neighborhood of $x_{0}$. Using this notion we consider the following modification of procedure (2.2)

$$
x_{n+1}=x_{n}-\Gamma\left(x_{0}\right) P x_{n}, \quad n=0,1,2, \ldots
$$

THEOREM 2.2. Suppose that there exists positive numbers $0<q<1$, $r, \eta$ and $B$ such that the uniform inverse derivative $\Gamma\left(x_{0}\right)$ satisfies the condition

$$
\text { (2.15) } \quad\|y\|^{-1}\left\|P\left(x+\Gamma\left(x_{0}\right) y\right)-P x-y\right\| \leqslant q \quad \text { for } x \in S\left(x_{0}, r\right)
$$

and $\quad\|y\| \leqslant \eta\left\|\Gamma\left(x_{0}\right)\right\| \leqslant B$ and $\left\|P x_{0}\right\| \leqslant \eta$.
Then there exists a solution $x^{*} \in S\left(x_{0}, r\right)$ and the sequence of $x_{n}$ determined by (2.14) converges toward $x^{*}$, i. e. $x_{n} \rightarrow x^{*}, P x^{*}=0, x^{*} \in S\left(x_{0}, r\right)$ and the error estimate (2.8) holds. If $\Gamma\left(x_{0}\right)$ is onto and (2.15) is satisfied for all $y \in Y$ such that $\left\|\Gamma\left(x_{0}\right) y\right\| \leqslant 2 r$, then the solution $x^{*}$ is unique in $S\left(x_{0}, r\right)$.

The proof of this theorem is exactly the same as that of Theorem 2.1.

3. Contractors. Analyzing the proof of Theorem 2.1 we can see that condition (2.3) plays the basic role in our argument. This observation leads to the concept of a contractor. Let $P: X \rightarrow Y$ be a nonlinar mapping and let $\Gamma(x): Y \rightarrow X$ be a bounded linear operator associated with $x$.

Definimion. We say $P$ has a contractor $\Gamma(x)$ if there is a positive number $q<1$ such that

$$
\|P(x+\Gamma(x) y)-P x-y\| \leqslant q\|y\|,
$$

where $x \in X$ and $y \in Y$ are to be adjusted to the problem.

For instance, if $P$ has ai Fréchet derivative $P^{\prime}(x)$ satisfying

$\left(3.1^{*}\right) \quad\left\|P^{\prime}(x) \Gamma(x) y-y\right\| \leqslant q^{\prime}\|y\|, \quad 0<q^{\prime}<1$

then $\Gamma(x)$ is a contractor and condition (3.1) is satisfied with $q=\left(1+q^{\prime}\right) / 2$ and $\|y\| \leqslant \delta\|\Gamma(x)\|^{-1}$, where $\delta$ is chosen so as to satisfy $\| P(x+h)-P x-$ $-P^{\prime}(x) h\left\|\leqslant\left(1-q^{\prime}\right) 2^{-1}\right\| h \|$ for $\|h\| \leqslant \delta$. Then for $h=\Gamma(x) y$ we have

$$
\|P(x+\Gamma(x) y)-P x-y\|
$$

$\leqslant\left\|P(x+\Gamma(x) y)-P^{\prime}(x) \Gamma(x) y\right\|+\left\|P^{\prime}(x) \Gamma(x) y-y\right\| \leqslant\left[\left(1-q^{\prime}\right) 2^{-1}+q^{\prime}\right]\|y\|$, and $\|y\| \leqslant \delta\|\Gamma(x)\|^{-1}$ implies $\|h\|=\|\Gamma(x) y\| \leqslant \delta$.

Obviously, an inverse derivative is a contractor. We say that $P: X \rightarrow Y$ has a bounded contractor $\Gamma(x)$ if $\|\Gamma(x)\| \leqslant B$ for all $x$ of a certain region.

Suppose now that $P$ has a contractor $\Gamma(x)$ satisfying condition (3.1) for all $y$ of $Y$. Then it is easily seen that the following inequality can be derived from (3.1)

$$
(1-q)\|y\| \leqslant\|P(x+\Gamma(x) y)-P x\| \quad \text { for } y \in Y .
$$

It follows from (3.1) that the contractor $\Gamma(x)$ is a one-to-one mapping and if $P$ is continuous, then (3.2) yields the continuity of the inverse $[\Gamma(x)]^{-1}$, i. e. then $\Gamma(x)$ is a homeomorphism of $Y$ onto a closed subspace of $X$. A contractor $\Gamma(x)$ is called regular, if (3.1) is satisfied for all $y \in X$ and $D(P)=\Gamma(x)(Y)$, where $D(P)$ is the domain of $P$. We say that $x$ is a regular point of $P$, if $P^{-1}(P x)=\{x\}$ and $P x_{n} \rightarrow P x$ implies $x_{n} \rightarrow x$.

LEMIMA 3.2. If a contractor $\Gamma(x)$ exists for $x \in D(P)$ and is regular, then $x$ is a regular point of $P$. If $\Gamma(x)$ is regular and onto, $i$. e. $\Gamma(x)(Y)=X$, then $X$ is a regular point of $P$ and $P$ is continuous at $x$. If $\Gamma(x)$ is regular for every $x \in D(P)$, then $P$ has a continuous inverse mapping $P^{-1}$. If $\Gamma(x)$ 
is regular and onto for every $x \in D(P)$, then $P$ is a homeomorphism of $X$ onto $P(X)$

Proof. The proof follows from inequality (3.2), since $x_{n} \in D(P)$ implies $x_{n}-x=\Gamma(x) y_{n}$ for some $y_{n} \in Y$. If $\Gamma(x)$ is onto, then the continuity of $P$ results from the continuity of the invers operator $[\Gamma(x)]^{-1}$ and from (3.1).

THEOREM 3.1. Theorem 2.1 remains true if we replace there the inverse derivative by a bounded contractor $\Gamma(x)$.

The proof is the same as that of Theorem 2,1. We say that the linear bounded operator $\Gamma\left(x_{0}\right): Y \rightarrow X$ is a uniform contractor of $P$ at $x_{0}$ if there exist positive numbers $0<q<1, r$ and $\eta$ such that condition $(2.15)$ is satisfied for $x \in S\left(x_{0}, r\right)$ and $\|y\| \leqslant \eta$. If the Fréchet derivative $P^{\prime}(x)$ exists and is Lipschitz continuous with constant $K$ in some neighborhood of $x_{0}$ and $P^{\prime}\left(x_{0}\right)$ is invertible, then $\Gamma\left(x_{0}\right)=\left[P^{\prime}\left(x_{0}\right)\right]^{-1}$ is a uniform inverse derivative, since

$$
\begin{aligned}
& \left\|P\left(x+\Gamma\left(x_{0}\right) y\right)-P x-y\right\|=\left\|P\left(x+\Gamma\left(x_{0}\right) y\right)-P x-P^{\prime}\left(x_{0}\right) \Gamma\left(x_{0}\right) y\right\| \\
& \leqslant\left\|P\left(x+\Gamma\left(x_{0}\right) y\right)-P x-P^{\prime}(x) \Gamma\left(x_{0}\right) y\right\|+\left\|P^{\prime}(x) \Gamma\left(x_{0}\right) y-P^{\prime}\left(x_{0}\right) \Gamma\left(x_{0}\right) y\right\| \\
& \leqslant 2^{-1} K\left\|\Gamma\left(x_{0}\right)\right\|^{2}\|y\|^{2}+K\left\|x-x_{0}\right\|\left\|\Gamma\left(x_{0}\right)\right\|\|y\| .
\end{aligned}
$$

Evidently, $\Gamma\left(x_{0}\right)$ is a uniform contractor.

THEOREM 3.2. Theorem 2.2 remains true if we replace there the uniform inverse derivative by the uniform contractor $\Gamma\left(x_{0}\right)$.

The proof of this theorem is exactly the same as that of Theorem 2.2.

4. Implicit function theorems using contractors. On the basis of Theorems 3.1 and 3.2 we can generalize the well known implicit function theorem. Let $X, Z$ and $Y$ be Banach spaces. Consider the operator $P(x, z): X \times Z \rightarrow Y$. Put $S=\left[(x, z):\left\|x-x_{0}\right\| \leqslant r,\left\|z-z_{0}\right\| \leqslant \varrho\right]$ and suppose that for every $z$ such that $(x, z) \in S, P$ has a contractor $\Gamma(x, z): X \rightarrow X$ which is strongly continuous in $(x, z)$.

THEOREM 4.1. Suppose that there exist positive numbers $0<q<1$, $r, \varrho, \eta$ and $B$ such that

(4.1) $\|P(x+\Gamma(x, z) y, z)-P(x, z)-y\| \leqslant q\|y\|$ for $(x, z) \in S$ and $\|y\| \leqslant \eta$.

$$
\begin{gathered}
\|\Gamma(x, z)\| \leqslant B \quad \text { for }(x, z) \in S . \\
\left\|P\left(x_{0}, z\right)\right\| \leqslant \eta . \\
B \eta(1-q)^{-1} \leqslant r
\end{gathered}
$$

(4.5) $P(x, z)$ is closed in $S$ for every fixed $z$ restrioted to $S$, i. e. $\left(x_{n}, z\right) \in S$, $x_{n} \rightarrow x$ and $P\left(x_{n}, z\right) \rightarrow y$ imply $y=P(x, z)$.
(4.6) $P(x, z)$ is continuous in $S$ with respect to $z$ for every fixed $x$ restricted to $S$.

$$
P\left(x_{0}, z_{0}\right)=0 \text {. }
$$

Then there exists a continuous function $x=g(z)$ such that $P(g(z), z)=0$, $(g(z), z) \in S$.

Proof. The proof is based on Theorem 3.1. Consider the iteration procedure $x_{n+1}(z)=x_{n}(z)-\Gamma\left(x_{n}, z\right) P\left(x_{n}, z\right)$ for $n=0,1,2, \ldots$ and every fixed $z$ restricted to $S$. In virtue of Theorem 3.1, the sequence of $x_{n}(z)$ converges to an element $x=g(z)$ such that $(x, z) \in S$ and $P(x, z)=0$. The functions $x_{n}(z)$ are continuous and it is easily seen that the convergence of $x_{n}(z)$ is uniform in $z$. Thus, $x(z)$ is continuous.

Remark 4.1. If $P(x, z)$ is jointly continuous in $(x, z)$, then conditions (4.1), (4.2) and (4.7) are sufficient for the theorem and numbers $r$, $\varrho$ can be chosen so as to satify (4.3) and (4.4). If $\Gamma(x, z)$ is onto, then the function $g(z)$ is unique in $S$ provided that condition (4.1) is satisfied for all $y \in Y$ such $\|\Gamma(x, z) y\| \leqslant 2 r$.

THEOREM 4.2. Theorem 4.1 remains true if we replace there the contractor $\Gamma(x, z)$ by a uniform contractor $\Gamma\left(x_{0}, z\right)$, i. e. if we replace condition (4.1) by

$$
\left\|P\left(x+\Gamma\left(x_{0}, z\right) y, z\right)-P(x, z)-y\right\| \leqslant q\|y\| .
$$

Remark 4.1 remains also true.

The proof is exactly the same as that of Theorem 4.1, but we use here the following iteration procedure

$$
x_{n+1}(z)=x_{n}(z)-\Gamma\left(x_{0}, z\right) P\left(x_{n}, z\right) \quad \text { for } n=0,1,2, \ldots
$$

5. A generalization of the Banach contraction principle. Oonsider the operator equation $P x=\xi$, where $P: D(P) \subset X \rightarrow Y$ is a closed nonlinear operator.

THEOREM 5.1. SUPpose that the closed operator $P$ has a bounded contractor $\Gamma(x)$ such that

$$
\|P(x+\Gamma(x) y)-P x-y\| \leqslant q\|y\| \text { for all } y \in Y,
$$

where $0<q<1$ and $\|\Gamma(x)\| \leqslant B$ for all $x \in D(P)$. Then the equation $P x=y$ has a solution for arbitrary $y \in \bar{Y}$. If $\Gamma(x)$ is regular (and onto) for every $x \in D(P)$, then the inverse $P^{-1}$ exists and is Lipschitz continuous with the constant $B(1-q)^{-1}$ (and $P$ is continuous).

Proof. For arbitrary fixed $\xi \in Y$ consider the operator determined by $P x-\xi$. This operator has the same contractor $\Gamma(x)$ and we apply the iteration procedure

$$
x_{n+1}=x_{n}-\Gamma\left(x_{n}\right)\left(P x_{n}-\xi\right)
$$


where $x_{0} \in D(P)$ is arbitrarily chosen. The sequence of $x_{n}$ converges to an element $x=R \xi$ and $P x_{n} \rightarrow \xi$ as $n \rightarrow \infty$. Since $P$ is closed, $P(R \xi)=\xi$.

Suppose now that $\Gamma(x)$ is regular. Let $P\left(R \xi_{1}\right)=\xi_{1}$ and $P\left(R \xi_{2}\right)=\xi_{2}$. Then we can write $R \xi_{2}=R \xi_{1}+\Gamma\left(R \xi_{1}\right) y$. Hence, using inequality (3.2) we obtain

$$
\left\|R \xi_{2}-R \xi_{1}\right\| \leqslant\left\|\Gamma\left(R \xi_{1}\right)\right\|\|y\| \leqslant B(1-q)^{-1}\left\|\xi_{2}-\xi_{1}\right\| .
$$

From Lemma 3.1 follows that if $\Gamma(x)$ is onto, then $P$ is continuous.

The global constant $B$ can be replaced by a local one $B\left(x_{0}, \xi\right)$ such that $\|\Gamma(x)\| \leqslant B\left(x_{0}, \xi\right)$ for $\left\|x-x_{0}\right\| \leqslant r$ and $B\left(x_{0}, \xi\right)\left\|P x_{0}-\xi\right\|(1-q)^{-1} \leqslant r$. Then we can prove only the continuity of $P^{-1}$.

Consider now equations of the second kind $P x=x-F x=\xi$, where $F: X \rightarrow X$. Condition (5.1) yields here

(5.3) $\quad\|F x-F(x+\Gamma(x) y)-(I-\Gamma(x)) y\| \leqslant q\|y\|$ for all $y \in X$,

where $I$ is the identity mapping.

THEOREM 5.2. Theorem 5.1 remains true for the equation $x-F x=\xi$ $x, \xi \in \mathcal{X}$, if we replace there condition. (5.1) by (5.3).

The proof follows from Theorem 5.1.

Remark 5.1. If $F: X \rightarrow X$ is a contraction with the constant $q<1$, then, obviously, a contractor $\Gamma(x)$, satisfying all conditions of Theorem 8 , exists and it is the identity mapping, i. e. $\Gamma(x)=I$. Thus, Theorem 5.2 generalizes the well known Banach contraction principle.

The following remark will be used in the next section.

Remark 5.2. Consider the equation

एक.

$$
P x=x-F(x)=\xi, \quad x, \xi \in X .
$$

Then Theorem 5.2 remains true if condition (5.3) is replaced by the following inequality

(5.5) $\|\tilde{F}(x+y+\Gamma(x) y)-F x-\tilde{\Gamma}(x) y\| \leqslant q\|y\|$, for all $y \in X$ and $0<q<1$, where $\|\tilde{\Gamma}(x)\| \leqslant B$.

For we replace in (5.2) the operator $I-\Gamma(x)$ by $\tilde{\Gamma}(x)$. If $(I+\tilde{\Gamma}(x))(X)$ $=D(F)$ for all $x \in D(F)$ then the inverse of $x-F(x)$ exists and is defined on the whole of $X$ and is Lipschitz continuous with the constant $(1+B) \times$ $\times(1-q)^{-1}$. The iteration procedure in this case is determined as follows

$$
x_{n+1}=x_{n}-\left(I+\tilde{\Gamma}\left(x_{n}\right)\right)\left(x_{n}-F x_{n}-\xi\right), \quad n=0,1,2, \ldots
$$

The initial approximate solution $x_{0}$ can be chosen arbitrarily and the procedure converges toward a solution. For equations of second kind it is convinient to have the contractor in the form $I+\tilde{T^{\prime}}(x), x \in D\left(F^{\prime}\right)$.
6. Nonlinear evolution equations, a generalization of the Piccard theorem in Banach spaces. Consider the initial value problem

$$
\frac{d x}{d t}=F(t, x), \quad 0 \leqslant t \leqslant T, x(0)=\xi,
$$

where the unknown $x=x(t)$ is a function defined on the real interval $[0, T]$ with values in the Banach space $X$, and $F:[0, T] \times X \rightarrow X$ is a continuous mapping. Instead of (6.1) we consider the integral equation

$$
x(t)-\int_{0}^{t} F(s, x(s)) d s=\xi .
$$

Denote by $X_{T}$ the space of all continuous functions $x=x(t)$ defined on $[0, T]$ with values in $X$ and with the norm $\|x\|_{d}=\max [\|x(t)\|: 0 \leqslant t$ $\leqslant T]$. Considering equation (6.2) as an operator equation in $X_{T}$ we can apply our generalization of the Banach contraction principle discussed in Section 5, especially Remark 5.2.

For arbitrary fixed $x \in X$ and $t \epsilon[0, T]$ let $\Gamma(t, x): X \rightarrow X$ be a bounded linear operator, strongly continuous with respect to $(t, x)$ in the sense of the operator norm. Suppose that there exist positive numbers $K, B$ such that the inequality

$$
\begin{gathered}
\max _{0 \leqslant t \leqslant T}\left\|F\left(t, x(t)+y(t)+\int_{0}^{t} \Gamma(s, x(s)) y(s) d s\right)-F(t, x(t))-\Gamma(t, x(t)) y(t)\right\| \\
\leqslant K\|y\|_{C}
\end{gathered}
$$

is satisfied for arbitrary continuous functions $x=x(t), y=y(t) \in X_{T}$, where $\|\Gamma(t, x)\| \leqslant B$ for all $x \in X$ and $t \in[0, T]$. Then we say that $F(t, x)$ has a bounded integral contractor $\left\{I+\int_{0}^{t} \Gamma\right\}$. A bounded integral contractor is said to be regular if the integral equation

$$
y(t)+\int_{0}^{t} \Gamma(s, x(s)) y(s) d s=z(t), \quad 0 \leqslant t \leqslant T
$$

has a continuous solution $y(t)$ for arbitrary fixed and continuous functions $x(t)$ and $z(t) \in X_{T}$. Obviously, if $F(t, x)$ satisfies Lipschitz condition uniformIy in $t$, than $\left\{I+\int_{0}^{t} \Gamma\right\}$, where $\Gamma \equiv 0$, is a regular bounded integral contractor.

THEOREM 6.1. Suppose that $F(t, z)$ has a bounded integral contractor and $T$ is such that $T K=q<1$. Then for arbitrary $\xi \in X$ equation (6.2) has a continuous solution $x(t)$. If the bounded integral contractor is regular then the solution $x \in X_{T}$ is unique and Lipschitz continuous with respect to $\xi$. 
Proof. Consider in $X_{T}$ the iteration procedure

$$
x_{n+1}=x_{n}-\left[y_{n}+\int_{0}^{t} \Gamma\left(s, x_{n}\right) y_{n} d s\right] \quad \text { for } n_{n}=0,1,2, \ldots,
$$

where $x_{n}=x_{n}(t), y_{n}=y_{n}(t)=x_{n}(t)-\int_{0}^{t} F\left(s, x_{n}(s)\right) d s-\xi$.

We have, by $(6.5)$

$$
y_{n+1}=\int_{0}^{t}\left[F\left(s, x_{n}\right)-F\left(s, x_{n}-y_{n}-\int_{0}^{s} T^{\top}\left(\tau, x_{n}\right) y_{n} d \tau\right)-\Gamma\left(s, x_{n}\right) y_{n}\right] d s
$$

Hence, replacing $y$ by $-y$ in (6.3), we obtain that $\left\|y_{n+1}\right\|_{\sigma} \leqslant q\left\|y_{n}\right\|_{d}$. Thus, the sequence of $y_{n}$ is convergent in $X_{T}$ toward zero. Since $\|\Gamma(t, x)\| \leqslant B$, it follows from (6.5) that $\sum_{n=0}^{\infty}\left\|x_{n+1}-x_{n}\right\|_{C} \leqslant(1+T B)\left\|y_{0}\right\|_{C}(1-q)^{-1}$, and that $x_{n}=x_{n}(t)$ is continuous for $n=0,1,2, \ldots$, where $x_{0}=x_{0}(t) \epsilon X_{T}$ can be arbitrarily chosen. Therefore, the sequence of $x_{n}$ has a limit $x \in \bar{X}_{n}$ which is a solution to (6.2). In the same way as in the proof of Theorem 5.2 , we derive from (6.4) that the solution $x$ is unique and Lipschitz continuous with respect to $\xi$.

It is interesting to observe that the contractor for (6.2) at each fixed $(t, x)$ is naturally defined as a linear integral operator of the same kind as the operator in the equation (6.2).

Theorem 6.1 generalizes the well known Piccard theorem for evolution equations in Banach spaces (see [7]). For as we mentioned above, if $F(t, x)$ is $t$-uniformly Lipschitz continuous, then with $\Gamma \equiv 0$ it satisfies the assumptions of Theorem 6.1.

7. A coincidence theorem and a generalization of Krasnoselskii's fixed point theorem. The purpose of this section is to show how to combine the contractor method and the Schauder [13] fixed point principle.

Let $W$ be a closed bounded convex set of a Banach space $X$. Given two operators acting in $X: P: D(P) \rightarrow X$ and $Q: W \rightarrow X$.

THEOREM 7.1. Suppose that $P$ is a elosed operator having a bounded regular contractor $\{\Gamma(x)\}$ satisfying the inequality

$$
\|P(x+\Gamma(x) y)-P x-y\| \leqslant q\|y\| \quad \text { with } 0<q<1
$$

$$
\text { for all } x \in D(P), y \in X \text {, }
$$

where $D(P)=\Gamma(x)(Y)$ and $\|\Gamma(x)\| \leqslant B$. Suppose that $Q$ is completely continuous and

(7.2) $\quad Q x-P y \in W$ and $\quad x+\Gamma(x) y \in W \quad$ for arbitrary $x, y \in W$.

Then $P$ and $Q$ have a coincidence point $x^{*} \in W$, i. e. $P x^{*}=Q x^{*}$.
Proof. On virtue of Theorem 5.1, for arbitrary $x \epsilon W$ the equation $P y=Q x$ has a unique solution $y$ which is a limit of the iteration procedure $x_{n+1}=x_{n}-\Gamma\left(x_{n}\right)\left[P x_{n}-Q x\right]$. It follows from (7.2) that $x_{n} \epsilon W$ for $n=0,1,2, \ldots$ Hence, $y \in W$ and $y=P^{-1} Q x$ is completely continuous, since the inverse $P^{-1}$ exists and is continuous, by Theorem 5.1. The Schauder fixed point theorem yields the existence of a point $x^{*} \epsilon W$ such that $x^{*}=P^{-1} Q x^{*}$, i. e. $P x^{*}=Q x^{*}$.

Consider now the case where $P x=x-F x$.

THEOREM 7.2. Suppose that $F: D(F) \rightarrow X$, is a closed operator having a bounded regular contractor $\{I+\Gamma(x)\}$ such that the inequality $\left\|F^{\prime}(x+\Gamma(x) y)-F^{\prime}(x)-\Gamma(x) y\right\| \leqslant q\|y\|, 0<q<1$ is satisfied for all $x \in D(F)$ and $y \in X$, where $\|\Gamma(x)\| \leqslant B$ and $D(F)=\Gamma(x)(Y)$. Suppose that $Q$ is completely continuous and

(7.3) $\quad F y+Q x \in W$ and $x+\Gamma(x)[y-x] \epsilon W \quad$ for arbitrary $x, y \in W$.

Then there exists a fixed point $x^{*} \in W$ such that $x^{*}=F x^{*}+Q x^{*}$.

Proof. On virtue of Theorem 5.2 and Remark 5.2, for arbitrary $x \in W$ the equation $y-F y=Q x$ has a unique solution $y$ which is a limit of the iteration procedure $x_{n+1}=x_{n}-\left[I+\Gamma\left(x_{n}\right)\right]\left[x_{n}-F x_{n}-Q x\right]$. Conditions (7.2) imply that $x_{n} \in W$ for $n=0,1,2, \ldots$ and, consequently, $y \in W$. It follows from the existence and continuity of the inverse $P^{-1}$ that $P^{-1} Q x$ is completely continuous, where $P x=x-F x$. Applying Schauder's fixed point theorem we obtain the existence of $x^{*} \in W$ such that $x^{*}-F x^{*}$ $=Q x^{*}$.

Both Theorems 7.1 and 7.2 can be considered as a generalization of the following theorem of Karsnoselskii [10]:

If $F$ is a contraction (i. e. Lipschitz continuous with a constant $q<1$ ) and $Q$ is completely continuous and $F y+Q x \in W$ for arbitrary $x, y \in W$, then there exists a fixed point $x^{*} \in W$ such that $x^{*}=F x^{*}+Q x^{*}$. If the assumptions of Krasnoselskii's theorem are satisfied, then by putting in Theorem $7.2 \Gamma(x) \equiv 0$ the identity mapping $I$ will be a bounded regular contractor satisfying conditions (7.2).

8. Stationary points of nonlinear functionals. Let $F: X \rightarrow R$ be a nonlinear functional differentiable in some sphere $S\left(x_{0}, r\right)$ of $X$ and denote by $F^{\prime}(x)$ the Fréchet derivative (gradient) of $F$ at $x$. The problem of unconstraint optimization of $F x$ reduces practically to finding stationary points $x$ of $F$, i. e. satisfying the equation

$$
F^{\prime} x=0, \quad x \in X \text {. }
$$

Considering $F^{\prime}: X \rightarrow X^{\prime}$ as a nonlinear (gradient) operator from the Banach space $X$ into its dual Banach space $X^{\prime}$, we can apply the contractor method to solve equation (8.1). 
THEOREM 8.1. Suppose that the gradient $F^{\prime}$ has a contractor $\Gamma(x): X^{\prime} \rightarrow X$ for every $x \in S\left(x_{0}, r\right)$ such that

(8.2) $\left\|F^{\prime}(x+\Gamma(x) y)-F^{\prime} x-y\right\| \leqslant q\|y\| \quad$ for $x \in S\left(x_{0}, r\right), y \in X^{\prime},\|y\| \leqslant \eta$, where $\|\Gamma(x)\| \leqslant B, \| F^{\prime}\left(x_{0} \| \leqslant \eta\right.$ and $B \eta(1-q)^{-1} \leqslant r$ for some constants $0<q<1, B$ and $\eta$. If $F^{\prime}$ is closed on $S\left(x_{0}, r\right)$, then it has a stationary point $x^{*} \in S\left(x_{0}, r\right), i$ e. $F^{\prime} x^{*}=0$, which is a limit of the sequenoe $\left\{x_{n}\right\}$ defined as follows $x_{n+1}=x_{n}-\Gamma\left(x_{n}\right) F^{\prime} x_{n}, n=0,1,2, \ldots$ and the error estimate is given by the formula $\left\|x_{n}-x^{*}\right\| \leqslant B q^{n}(1-q)^{-1}$.

Proof. The proof is exactly the same as that of Theorem 2.1.

Remark 8.1. If the contractor is additionally onto and satisfies (8.2) for all $y \in X^{\prime}$ such that $\|\Gamma(x) y\| \leqslant 2 r$ for $x \in S\left(x_{0}, r\right)$, then $I F$ has in $S\left(x_{0}, r\right)$ a unique stationary point $x^{*}$.

This assertion follows from the argument in Remark 2.1.

Remark 8.2: It is sufficient to assume that $F^{\prime}$ is defined on a subset $D\left(F^{\prime}\right)=S\left(x_{0}, r\right) \cap \Gamma(x)\left(X^{\prime}\right)$, where the intersection is the same for all $x \in D\left(F^{\prime}\right)$ and $x_{0} \in D\left(F^{\prime}\right)$. In this case the uniqueness for $x^{*}$ follows if condition (8.2) holds for all $y \in X^{\prime}$ such that $\|\Gamma(x) y\| \leqslant 2 r, x \in D\left(F^{\prime}\right)$.

\section{method.}

9. Various iteration procedures as special cases of the contractor

9.1. It is shown in Section 2. that the Newton-Kantorovich method (2.11) is a special case of the contractor method and the same is true for the generalization of Newton's method (2.13) for nonlinear functionals. The following modification of the Newton-Kantorovich method is given by Bartle [4].

$$
x_{n+1}=x_{n}-\left[P^{\prime}\left(z_{n}\right)\right]^{-1} P x_{n}
$$

provided that the initial guess $x_{0}$ and the arbitrarily selected points $z_{n}$ are sufficiently close to the solution desired. It is easy to see that the contractor for the method (9.1) is $\Gamma(x)=\left[P^{\prime}(x)\right]^{-1}$. Under the assumptions made in [4] the contractor satisfies the following inequality:

$$
\left\|P\left(x+\left[P^{\prime}(z)\right]^{-1} y\right)-P x-y\right\| \leqslant(1 / 2 \lambda)\left\|\left[P^{\prime}(z)\right]^{-1} y\right\| \leqslant(1 / 2 \lambda) \lambda\|y\|,
$$

where $\lambda$ and $\beta$ are chosen so as to satisfy $\left\|\left[P^{\prime}(z)\right]^{-1}\right\|<\lambda$ if $\left\|z-x_{0}\right\| \leqslant \beta$, $\left\|x-x_{0}\right\| \leqslant \beta$ and. $\left\|x+\left[P^{\prime}(z)\right]^{-1} y-x_{0}\right\| \leqslant \beta$ (see Lemma, $2[4]$ ).

9.2. It is shown in [3] that the method of steepest descent developed by Kantorovich [6], the minimum residual method investigated by Krasnoselskii and Krein [9] and other gradient methods (see [2], [8], [10]) are special cases of Newton's method (2.13) for nonlinear functionals. Thus, these methods can'also be consiclered as special cases of the contractor method. This is the case from variational point of view, i. e. when we reduce the operator equation to the minimum problem of a non-negative nonlinear functional $F$, where for instance, $F(x)=\|P x\|^{2}=0$ is required. However, we can see that these and other methods can also be considered as contractor methods in the direct sense of (2.2).

9.3. Let $A: H \rightarrow H$ be a linear self-adjoint and positive definite operator in the real Hilbert space $H$ such that $m(x, x) \leqslant(A x, x) \leqslant M(x, x)$, where $0<\mathrm{m}<M<\infty$. Consider the equation

$$
A x=b, \quad x, b \in H .
$$

The operator $P x=A x-b$ is differentiable in the sense of Fréchet and $P^{\prime}(x)=A$. It is easy to verify that $\|\alpha A-I\|<1$ if $0<\alpha<2 / M$. Thus, putting in $\left(3.1^{*}\right) \Gamma(x)=\alpha I$ we obtain a contractor and the corresponding contractor method will be the method of successive approximation with parameter $\alpha$ :

$$
x_{n+1}=x_{n}-\alpha\left(A x_{n}-b\right), \quad n=0,1,2, \ldots
$$

Replacing in (9.3) a by $\alpha(x)=(r, A r) /(A r, A r)$, where $r=r(x)=A x-b$ we obtain the minimum residual method investigated by Krasnoselskii and Krein [9]:

$$
\begin{array}{r}
x_{n+1}=x_{n}-\alpha_{n} r_{n}, n=0,1,2, \ldots, \quad \text { where } \alpha_{n}=\alpha\left(x_{n}\right) \text { and } \\
r_{n}=r\left(x_{n}\right) .
\end{array}
$$

The contractor here is $\alpha(x) I$ and the contractor inequality (3.1) yields in this case

$$
\|A(x+\alpha(x) y)-A x-y\| \leqslant(M-m)(M+m)^{-1}\|y\| .
$$

This inequality is satisfied for $y=r(x)$, in virtue of the following inequality (see [10] p. 109)

$$
\|A(x-\alpha(x) r(x))-b\| \leqslant(M+m)(M-m)^{-1}\|r(x)\| .
$$

To prove last inequality let us observe that

$$
\|A(x-\alpha(x) r(x))-b\|^{2}=\min _{t} \| A\left(x-\operatorname{tr}(x)-b\left\|^{2}=\min _{t}\right\| r(x)-t A r(x) \|^{2} .\right.
$$

But for $t=2(M+m)^{-1}$ we have $\left\|I-\frac{2}{M+m} A\right\|=(M-m)(M+m)^{-1}$ and, consequently, we obtain (9.5).

9.4. The method of steepest descent developed by Kantorovich [6] for soloving (9.2) is defined as follows:

$$
x_{n+1}=x_{n}-\beta_{n} r_{n}, \quad \text { where } \beta_{n}=\left(r_{n}, r_{n}\right)\left(A r_{n}, r_{n}\right)^{-1} \text {. }
$$


Consider the Hilbert space $H_{4}$ obtained from $H$ by introducing a new scalar product $[u, v]=\left(A^{-1} u, v\right), u, v \in H$. Then it is clear that the steepest descent method (9.6) is the minimum residual method (9.4) considered in the Hilbert space $H_{A}$. Thus, the steepest descent method (9.6) is a contractor method in the Hilbert space $H_{A}$. It is easily seen that $A$ is self adjoint and positive definit in $H_{A}$.

9.4. Consider the nonlinear operator equation

$$
P x=0, \quad \text { where } P: S\left(x_{0}, r\right) \rightarrow H,
$$

$P$ being continuously differentiable in the sense of Fréchet in the sphere $S\left(x_{0}, r\right) \subset H$ and $P^{\prime}(x)$ satisfies the inequality

$$
\text { (9.8) } \quad\left\|P^{\prime}(x) y\right\| \geqslant B^{-1}\|y\| \quad \text { for all } x \in S\left(x_{0}, r\right), y \in H \text {. }
$$

The following iteration procedure [2] is also a contractor method:

$$
x_{n+1}=x_{n}-\left\|P x_{n}\right\|^{2}\left\|Q\left(x_{n}\right)\right\|^{-2} 2^{-1} Q\left(x_{n}\right), \quad n=0,1, \ldots,
$$

where $Q(x)=\left[P^{\prime}(x)\right]^{*} P x\left(^{*}=\right.$ adjoint). Since $P$ is differentiable, we can expect that $\Gamma(x)=\|P x\|^{2}\|Q(x)\|^{-2} 2^{-1}\left[P^{\prime}(x)\right]^{*}$ will be a contractor, in virtue of $\left(3.1^{*}\right)$. Condition (9.8) implies that $\|\Gamma(x)\|$ is bounded for $x \in S\left(x_{0}, r\right)$. The existence of a solution of (9.7) as well as the convergence of (9.9) to this solution can be obtained from Theorem 3.1. It follows from the assumptions made in [2] that Theorem 3.1 can be applied. It is not difficult to see that the hypotheses made by Kivistik (see [1.0] p. 156) are also sufficient in order to apply the contractor method to his procedure

$$
x_{n+1}=x_{n}-\left(P^{\prime}\left(x_{n}\right) P x_{n}, P x_{n}\right)\left\|P^{\prime}\left(x_{n}\right) P x_{n}\right\|^{-2} P x_{n}, \quad n_{n}=0,1,2, \ldots
$$

Note that other procedures similar to $(9.9)$ can also be put in the unified scheme of the contractor method.

Remark 9.1. In all theorems which are global in nature, the requirement that the contractor $\Gamma(x)$ is bounded, i. e. $\|\Gamma(x)\| \leqslant B$ for $x \in D(P)$, can be replaced by the assumption that $\Gamma(x)$ is Lipschitz continuous with constant $K$.

Proof. We have, by (2.2),

$\left.\left\|\Gamma\left(x_{n+1}\right)\right\| \leqslant\left\|\Gamma\left(x_{n}\right)\right\|\left(1+K\|\| P x_{n} \|\right) \leqslant\left\|\Gamma\left(x_{n}\right)\right\|\left(1+K q^{n}\left\|P x_{0}\right\|\right) \leqslant \| \Gamma x_{n}\right) \|(1+\tau)$ for large $n$. Hence

$$
\left\|\Gamma\left(x_{n+1}\right)\right\|\left\|P x_{n+1}\right\| \leqslant(1+\tau) q\left\|\Gamma\left(x_{n}\right)\right\|\left\|P x_{n}\right\|, \quad \text { where } \tau<q^{-1}-1 .
$$

Then $P^{-1}$ will be continuous but not Lepschitzian.

An application of the contractor idea to Banach algebras will be given in a separate paper.

\section{Reference}

[1] M. Altman, Concerning approximate solutions of non-linear functional equations, Bull. Acad. Polon. Sci. Cl. III, 5 (1957), pp. 461-465.

[2] - On the approximate solutions of operator equations in Hilbert space, ibid. 15 (1957), pp. 605-609.

[3] - Oonnection between gradient methods and Newton's method for functionals, ibid. 9 (1961), pp. $877-880$.

[4] R. G. Bartle, Newton's method in Banach spaces, Proc. Amer. Math. Soc. 6 (1955), pp. 827-831.

[5] L. Collatz, Functional analysis and numerical mathematics, New York 1966

[6] L. V. Kantorovich and G. P. Akilov, Functional analysis in normed spaces, New York 1964

[7] T. Kato, Nonlinear evolution equations in Banach spaces, Proc. Symp. Appl Math., 17, pp. 50-67, Am. Math. Soc., Providence 1965.

[8] L. Kivistik, On a class of iteration processes in Hilbert space (Russian), Tartu Riikl. Üe. Toimetised 129 (1962), 365-381.

[9] M. A. Krasnoselskii and S. G. Krein, Iteration process with minimum residua (Russian), Matem. Sbornik (NS), 31 (1952), pp. 315-334.

[10] - G. M. Vainikko, P. P. Zabreiko, Ya. B. Rutitzkii and B. Ya. Stetzenko, Approximate solution of operator equations, (Russian) Nauka, Moscow 1969,

[11] M. Z. Nashed, Differentiability and related properties of nonlinear operators Some aspects of the role of differentials in nonlinear functional analysis in: Nonlinear functional analysis and applications, edit. by Louis B. Rall, New York-London 1971.

[12] J. M. Ortega and W. C. Rhein bold t, Iterative solutions of nonlinear equations in several variables, New York and London 1970.

[13] J. Schauder, Der Fixpunktsatz in Funtetionalräumen, Studia Math. 2 (1930), 171-180.

LOUISIANA STATE UNIVERSITY 